\title{
TECNOLOGIAS NÃO INVASIVAS DE CUIDADO NO PARTO REALIZADAS POR ENFERMEIRAS: A PERCEPÇÃO DE MULHERES
}

\author{
Non-invasive tecnologies of care at childbirth used by nurses: the perception of users' \\ women.
}
Tecnologías no-invasivas de cuidados en lo parto utilizadas por las enfermeras: la percepción de las mujeres usuarias.

Natália Magalhães do Nascimento ${ }^{1}$

Thalita Rocha de Oliveira ${ }^{4}$

\author{
Jane Márcia Progianti
}

Octávio Muniz da Costa Vargens ${ }^{5}$

\section{RESUMO}

A pesquisa de natureza qualitativa, tipo descritiva, teve como objetivo identificar as atitudes e práticas de enfermeiras obstétricas e discutir seus efeitos durante o trabalho de par to na percepção de mulheres, atendidas em uma casa de parto. Fizeram parte do estudo 12 mulheres, tendo como instrumento de coleta de dados uma entrevista semiestruturada. A análise dos dados evidenciou que as mulheres reconheceram a atitude carinhosa e práticas como a livre movimentação corporal e o estímulo à presença de um acompanhante como as principais tecnologias não invasivas utilizadas durante o trabalho de parto. Quanto aos seus efeitos, as mulheres perceberam que as tecnologias favoreceram seus potenciais internos para tomada de decisões e identificaram as atitudes e práticas das enfermeiras como decisivas para que não desanimassem durante o parto. A postura e o uso pelas enfermeiras de tecnologias não invasivas contribuem para uma melhor percepção das mulheres sobre o seu processo de parto. Palavras-chave: Enfermagem Obstétrica. Humanização do Parto. Parto Normal.

\begin{abstract}
The research of qualitative nature, descriptive type had as objective to identify the obstetric nurses' practices and to discuss their effects during the childbirth, under the women's optics. Twelve women had been part of the study, having as instrument of collect of data a semi structured interview. Data analysis showed that women recognized the affectionate attitude, the corporal movement and the presence of companion as the main non-invasive technologies used during the childbirth work, in the House. As for their effects, the women realized that technologies favored their internal potentials at the time of making their own decisions. Besides, they identified the nurses' practices of the House as determinants for them have not discouraged during the childbirth work. The posture and the use by the nurses of non-invasive technologies contribute to a better perception of the women about her childbirth's process.
\end{abstract}

keywords: Obstetric nursing. Humanizing Delivery. Normal childbirth.

\section{Resumen}

La investigación de un estudio cualitativo, descriptivo, tuvo como objetivo identificar las prácticas de las enfermeras obstétricas y discutir sus efectos durante el trabajo del parto bajo de la perspectiva de las mujeres. Los participantes del estudio fueron doce mujeres, teniendo como instrumento de recolecta de datos una entrevista semi-estructurada. El análisis de los datos evidenció que las mujeres reconocieron la actitud cariñosa, el movimiento corporal y la presencia de un acompañante como las principales tecnologías no-invasivas utilizadas durante el trabajo de parto en la casa de nacimiento. Cuánto a sus efectos, las mujeres se dieron cuenta de que las tecnologías favorecían sus potenciales internos en la hora de tomar sus propias decisiones. Además de eso, identificaron las prácticas de las enfermeras de la casa de nacimiento como determinantes para que ellas no tengan desalentado durante el trabajo de parto. La actitud de las enfermeras y el uso de tecnologías no invasivas contribuyen a una mejor percepción de las mujeres acerca de su proceso de parto.

Palabras clave: Enfermería obstétrica. Parto Humanizado. Parto normal.

\footnotetext{
'Enfermeira Obstétrica, Mestranda da Faculdade de Enfermagem da UERJ.Rio de Janeiro-RJ-Brasil. E-mail: natymnenf@yahoo.com.br, ${ }^{2}$ Enfermeira Obstétrica, Doutora, Professora Adjunta do Departamento Materno-Infantil da Faculdade de Enfermagem da UERJ,Rio de Janeiro-RJ-Brasil. E-mail: jmprogi@uol.com.br, ${ }^{3}$ Enfermeira Obstétrica, Professora Substituta da Faculdade de Enfermagem da UERJ,Rio de Janeiro-RJ-Brasil. E-mail: rachelli_iozzi@yahoo.com.br, ${ }^{4}$ Enfermeira Obstétrica, Mestranda da Faculdade de Enfermagem da UFF,Rio de Janeiro-RJ-Brasil. E-mail: oliveira.thalita@oi.com.br,5Enfermeiro Obstétrico, Doutor, Professor Titular do Departamento Materno-Infantil da Faculdade de Enfermagem da UERJ, Brasil.Rio de Janeiro-RJ-E-mail: omcvargens@uol.com.br
} 


\section{INTRODUÇ̃̃O}

No Brasil, até meados dos anos 80 do último século, as políticas nacionais voltadas para a saúde da mulher eram elaboradas para atender demandas relativas à gravidez e ao parto. Elas traduziam uma visão sobre a mulher, baseada em sua especificidade biológica de reprodução, o que refletia, á época, o papel social de mãe, esperado da mulher pela sociedade brasileira.

No entanto, nos anos 70 , os governos militares consentiram a participação de entidades privadas e estrangeiras na distribuição de contraceptivos e na realização de laqueaduras tubárias, favorecendo investimentos internacionais que disponibilizavam métodos e técnicas "modernas" de contracepção dirigidas às populações pobres. Tal fato contribuiu com a hospitalização do parto e elevação das taxas de cesáreas, sem indicação clínica e com a finalidade de reduzir o crescimento populacional que ocorria nos países subdesenvolvidos e nas populações pobres.

A partir desses acontecimentos, o movimento feminista mundial iniciou uma crítica às desigualdades nas relações sociais entre homens e mulheres, que se traduziam em problemas de saúde e afetavam particularmente a população feminina. Reivindicou, portanto, a condição feminina de sujeitos de direito, com necessidades que iam além do momento da gestação e parto. Considerando as condições sociais, econômicas, culturais e afetivas, demandou ações que contemplassem as particularidades dos diferentes grupos populacionais.

Em meados dos anos 80, o movimento feminista brasileiro e o movimento sanitário, inseridos no contexto mundial da luta pelos direitos humanos, criticaram o modelo de saúde vigente e tiveram grande contribuição para a formulação do Programa de Assistência Integral à Saúde das Mulheres (PAISM) e para a reforma sanitária brasileira com princípios básicos de universalidade, equidade e integralidade.

Assim, em 1984, o PAISM ocorreu não só sob a perspectiva de resolver o impacto das desigualdades de gênero na saúde da mulher (atendendo princípios da reforma sanitária que embasaria a criação do Sistema Único de Saúde (SUS), mas também para questionar o saber médico no campo obstétrico que, com práticas obstétricas intervencionistas, transformou 0 parto em um ato médico.

No Brasil, a iniciativa de questionar a conduta dos profissionais de saúde no campo obstétrico, nos anos 90, deu início ao movimento de humanização do parto e do nascimento. Entende-se por humanização o aumento do grau de corresponsabilidades na produção de saúde, e as mudanças da atenção dos profissionais aos usuários e de gestão de seus processos de trabalho. Para isso, a humanização fundamentase nas seguintes ideias: troca e construção de saberes, trabalhos em equipe e consideração aos interesses dos diferentes atores do campo da saúde. ${ }^{1}$
Deste modo, o conceito de atenção humanizada ao parto envolve um conjunto de conhecimentos, práticas e atitudes que visam à promoção do parto e do nascimento saudáveis e a prevenção da morbimortalidade materna e perinatal. ${ }^{2}$ Nessa concepção, a humanização tem como foco a qualificação da atenção, envolvendo o respeito e a promoção dos direitos humanos, sexuais e reprodutivos, e, por outro, a formação de profissionais que implantem práticas baseadas nas evidências científicas em ambientes com instalações físicas adequadas e recursos tecnológicos disponíveis. ${ }^{3}$

Em meados dos anos 90, muitas enfermeiras obstétricas incorporaram em seu fazer práticas obstétricas recomendadas pela Organização Mundial de Saúde (OMS) e consideradas pelo Ministério da Saúde (MS) brasileiro como apropriadas. Deste modo, estas especialistas da enfermagem tiveram fundamental importância para a reconfiguração do campo obstétrico com a perspectiva da humanização do parto. ${ }^{4}$

Á época, especificamente na cidade do Rio de Janeiro, a aquisição destas práticas pela enfermeira obstétrica foi um dos motivos para que ocupassem espaços na assistência ao parto hospitalar e para que viabilizassem a criação e consolidação da Casa de Parto David Capistrano Filho. Até os dias de hoje, as enfermeiras obstétricas lutam para ocuparem e manterem-se nesses espaços, ${ }^{5}$ e neles vem desenvolvendo tecnologias que denominamos de tecnologias não invasivas de cuidado de enfermagem obstétrica. 0 conceito de tecnologia apresenta-se em três tipos de classificações: em tecnologias leves, que implicam a criação de relação entre sujeitos (profissional de saúde e cliente), e pode se concretizar através da comunicação, do acolhimento e vínculo. As leves-duras, que são os saberes bem estruturados que atuam no processo de saúde (por exemplo, a clínica médica e a epidemiologia), e as duras que são os equipamentos tecnológicos (como as máquinas, normas, rotinas e estruturas organizacionais). ${ }^{6}$

0 conceito de tecnologia também envolve saberes e habilidades que precisam ser distinguidas de equipamento ou aparelho. ${ }^{7}$ Com enfoque em saúde, pode, então, ser compreendida como um saber estruturado aplicado com intencionalidade e justificativa e que produz um resultado que satisfaça as necessidades individualizadas dos seres humanos. ${ }^{8}$

Neste contexto, o uso de tecnologias compreende a combinação de suas dimensões - leve, leve-dura e dura ${ }^{6}$ - com destaque para a potencialização do saber voltado à elaboração e desenvolvimento de práticas de cuidados relacionados aos processos de gestar e parir que não sejam invasivas à fisiologia do corpo, da mente e privacidade do ser feminino. 0 caráter não invasivo das tecnologias de cuidado da enfermagem obstétrica reside em acreditarmos que, quando o sujeito estabelece um vínculo de confiança com o profissional, ambos compartilham as decisões no planejamento dos seus cuidados. Portanto, mesmo quando os procedimentos tocam partes íntimas do corpo biológico ou do corpo sociocultural da mulher, 
estes não são percebidos como invasão da privacidade, da fisiologia do corpo ou mesmo da mente.

Assim, as tecnologias de cuidado de enfermagem obstétrica são definidas como o conjunto de técnicas, procedimentos e conhecimentos utilizados pelo enfermeiro durante sua relação de cuidado profissional, que, por sua concepção ecológica, compreende o parto como um processo fisiológico, respeitando sua natureza e a integridade corporal e psíquica das mulheres. ${ }^{4}$

Nessa concepção, a enfermeira obstétrica, ao implantar suas práticas obstétricas nos serviços de saúde com a proposta de humanização recomendada pela OMS, coloca à disposição das usuárias um conhecimento profissional específico, caracterizado por ser essencialmente relacional e derivado de um saber estruturado da enfermeira. Também é aberta porque integra saberes populares e de diversas disciplinas na construção do cuidado. Por ter como instrumentos básicos os corpos, proporciona conforto e autonomia ao incentivar as mulheres a reconhecerem e desenvolverem suas próprias habilidades. ${ }^{9}$

Diante do exposto, esse estudo visa identificar as práticas das enfermeiras obstétricas e discutir seus efeitos durante o trabalho de parto sob a ótica das mulheres.

A partir dessas reflexões, acreditamos que o estudo é relevante porque evidencia as tecnologias como instrumentos de cuidado utilizados pelas enfermeiras obstétricas e, ao mesmo tempo, a partir da percepção da mulher, traz evidências de seus efeitos.

\section{MÉTODO}

É um estudo descritivo com abordagem qualitativa porque se preocupa com o nível de realidade que não pode ser quantificado, trabalhando com o universo de significados, motivos, aspirações, crenças, valores e atitudes, correspondendo a um espaço mais profundo nas relações.

0 cenário da pesquisa foi uma casa de parto situada na zona oeste da cidade do Rio de Janeiro. Essa casa funciona como uma unidade de administração pública municipal que tem como um de seus propósitos resgatar a vivência do parto fisiológico, individualizado e com a participação ativa da mulher, possibilitando que esta experiência seja vivenciada pelos familiares. Sua equipe é composta por 18 enfermeira(o)s obstétrica(o)s, estando 2 na função de direção, 8 auxiliares de enfermagem e 2 assistentes sociais, com carga horária de 40 horas semanais. Também conta com uma equipe de administração, conservação e limpeza, bem como seguranças e motoristas de ambulância.

No período de setembro a outubro de 2007 , o projeto foi encaminhado ao Comitê de Ética, foi analisado e aprovado, recebendo o protocolo número 138/07.
Os sujeitos da pesquisa foram 12 mulheres que pariram nesta instituição entre os meses de janeiro a março de 2008. Elegemos as participantes com parto anterior em instituição hospitalar e que pariram pela primeira vez em uma casa de parto.

A coleta de dado foi realizada no período de março a maio de 2008, por meio de entrevistas semiestruturadas que seguiram um roteiro com perguntas relacionadas aos partos anteriores (inclusive o último ocorrido na casa de parto) e sobre o uso das tecnologias utilizadas neste último parto. Durante as entrevistas, pedimos para que descrevessem como foi o trabalho de parto. A partir de suas respostas e com as bases conceituais de tecnologias, pudemos identificar o que foi proposto para o estudo.

Neste estudo foram respeitados os preceitos éticos, conforme Resolução n 196 , aprovada pelo Conselho Nacional de Saúde em 10 de outubro de 1996. A autorização dos sujeitos para participação do estudo foi registrada através de um Termo de Consentimento Livre e Esclarecido, previamente apresentado às mulheres juntamente com os objetivos do estudo e a certificação de anonimato das informaç̃̃es. Os depoimentos foram gravados em aparelho de MP3 e, posteriormente, transcritos na íntegra.

0 método de análise seguiu as diretrizes da análise de conteúdo $^{10}$ que consiste em um conjunto de técnicas de comunicação com o intuito de obter, por meio de procedimentos sistemáticos e objetivos, a descrição dos conteúdos das mensagens, indicadores que permitem a inferência de conhecimentos relativos às condições de produção/recepção (variáveis inferidas) destas mensagens.

Assim, a análise do material coletado ocorreu em três etapas:

Ordenação dos dados: transcrição das gravações, releitura do material e organização dos depoimentos;

Classificação dos dados: reconhecimento e identificação das unidades de registro e agrupamento destas em unidades temáticas. Deste processo surgiram as seguintes categorias de análise: as práticas utilizadas pelas enfermeiras e reconhecidas pelas mulheres e 0 efeito dessas atitudes $\mathrm{e}$ práticas percebido pelas mulheres durante $\mathrm{o} \mathrm{trabalho} \mathrm{de} \mathrm{parto.}$

Análise final: articulação dos dados com os conceitos discutidos no estudo.

\section{RESULTADOS E DISCUSSÃO}

Neste estudo, as atitudes e práticas utilizadas pelas enfermeiras e reconhecidas pelas mulheres foram: a abordagem carinhosa, a movimentação corporal e a presença de um acompanhante.

\section{A abordagem carinhosa favorecendo 0 acolhimento}

As mulheres ressaltaram, durante toda a entrevista, que foram bem cuidadas. Perceberam uma abordagem 
carinhosa das enfermeiras obstétricas ao se relacionarem com elas:

Aqui elas me trataram muito bem! (Ent.05).

Omodo deles te tratarem [...] todos com muito carinho! (Ent.08).

O usuário dos serviços de saúde, em geral, não reclama da falta de conhecimento tecnológico no seu atendimento, mas sim da falta de interesse e de responsabilização dos diversos serviços em torno de si e de seu problema. No campo da saúde, o objeto não é a cura ou a promoção e proteção à saúde, mas a promoção do cuidado que, de fato, representa os reais objetivos do setor saúde. ${ }^{6}$ A postura de cuidar dos profissionais de saúde perante os usuários tem muito significado, à medida que eles se sentem valorizados e atendidos em suas necessidades.

Portanto, a abordagem carinhosa reconhecida pelas usuárias pode criar um vínculo entre o serviço e o usuário, 0 que atende a um dos princípios do acolhimento. ${ }^{11}$ Essa concepção está fundamentada na ideia de cuidado como um processo que envolve ações, atitudes e comportamentos que buscam a promoção, manutenção e/ou recuperação da saúde, dignidade e totalidade humana. ${ }^{12}$ Para isso, o profissional deve incorporar em seu cuidado práticas que contribuam para que a mulher vivencie o processo do parto de maneira prazerosa.

Isso leva-nos a refletir que promover uma assistência humanizada é saber ouvir as parturientes e suas necessidades, valorizando sua história de vida, incluindo seus aspectos sociais, psicológicos e emocionais, que podem influenciar de modo significativo sua vivência no parto normal. Para isso, os profissionais de saúde que se propõem a acompanhar essas mulheres nesse momento devem acolhê-las, reconhecendo a importância da comunicação em sua prática.

0 acolhimento é um aspecto importante e essencial da política de humanização que implica em uma recepção humana e atentiva, na qual as queixas, preocupações, angústias e dúvidas das mulheres são ouvidas e lhes é garantida a responsabilidade por parte da equipe na resolubilidade dos problemas identificados, articulando-se com outras unidades de saúde para dar a continuidade à assistência, caso se faça necessário. ${ }^{11}$

\section{A movimentação corporal favorecendo 0 protagonismo}

Muitas mulheres reconheceram o estímulo à movimentação corporal como uma prática da enfermeira obstétrica. A movimentação corporal foi estimulada nas seguintes situações: quando a cliente foi encaminhada ao banho de imersão, quando a massagearam e estimularam a realização de alguns exercícios físicos:

Também fui pra banheira. Fizeram massagem. Fiquei naquela barra de ferro me agachando, fazendo exercício, depois fiquei caminhando (Ent. 09).
Essas práticas das enfermeiras abrangem um conjunto de cuidados que têm como objetivo oferecer à mulher a possibilidade de vivenciar a experiência de parir como um evento fisiológico, favorecendo o seu protagonismo que pode ser feito através do estímulo ao seu empoderamento.

No campo obstétrico, o processo de empoderamento da mulher traz uma nova concepção de poder, assumindo novas formas de responsabilidades coletivas, de tomada de decisões e de responsabilidades compartilhadas, ${ }^{13}$ quando envolve não só a consciência individual, mas uma ação coletiva com 0 propósito de alcançar as transformações sociais. Implica também a eliminação do raciocínio clínico médico como única alternativa, passando a enfermeira a cuidar em uma perspectiva de encorajamento das mulheres a serem as protagonistas do evento do parto e nascimento. ${ }^{4-14}$

\section{0 favorecimento da presença de acompanhante promovendo a tranquilidade e a segurança}

As mulheres mencionaram que a presença de acompanhante foi muito positivo para elas:

Vocêse sente mais segura... porque quando tive a minha primeira filha, eu nunca tinha ficado num hospital, e quando eu me vi ali sozinha, sem ninguém, entrei em desespero. Mas aqui, não. Aqui me senti mais tranquila com eles perto de mim [...]. Sei lá. Achei que foi muito legal. (Ent. 06).

Ao mencionarem a importância do acompanhante, as depoentes também reconheceram que o estímulo à presença $\mathrm{e}$ à participação ativa desta pessoa no seu processo de parir é uma prática muito utilizada pela enfermeira obstétrica. Neste sentido, quando o profissional integra um membro da família escolhido pela mulher, principalmente o pai do bebê, durante o trabalho de parto, está contribuindo para a parturiente se sentir mais confiante. Essa prática também favorece a humanização da assistência, além de ser uma prática baseada em evidências científicas.

0 papel do acompanhante é definido como elemento fundamental para dar suporte emocional. É uma das maneiras de a mulher encontrar forças para levar o trabalho de parto e parto de forma mais tranquila, diminuindo a ansiedade, e, assim, tornar o nascimento o mais "natural" possível..$^{15}$

Portanto, o acompanhante proporciona à mulher maior segurança e conforto durante 0 trabalho de parto e parto. Também tem contribuído para a redução de taxas de cesariana, duração do trabalho de parto, incentivo ao aleitamento materno, além de desenvolver na parturiente uma percepção positiva desse processo.

Tudo isso leva-nos a acreditar que estimular e facilitar a presença do acompanhante durante o trabalho de parto também é uma prática da enfermeira obstétrica que pode ser benéfica para a mulher na medida em que proporciona a ela maior 
tranquilidade e segurança, já que ela está compar tilhando com alguém de sua confiança um momento muito especial, como 0 nascimento do seu filho.

\section{0 efeito percebido pelas mulheres das práticas utilizadas pelas enfermeiras obstétricas em seu processo de parto}

Essa categoria surgiu a partir da descrição pelas entrevistadas dos efeitos das práticas e atitudes das enfermeiras durante seu trabalho de parto e parto. Estes foram reconhecidos de forma benéfica e estimulante.

Ao estudar essa categoria, evidenciamos nos discursos das entrevistadas um reconhecimento de si mesmas como mulheres de decisões e que possuíam potenciais internos:

Eu só fiz porque eu quis. Eu acho que isso é uma coisa que parte da gente [...] (Ent.11).

Essa impressão citada por elas pode ter sido influenciada por sentirem-se respeitadas nos seus direitos de escolha, durante o último trabalho de parto:

Elas dão o direito pra gente escolher, entendeu? E eu achei que foi muito legal (Ent. 12).

Observamos também que essas mulheres perceberam, nas práticas e atitudes no cuidado proporcionado pelas enfermeiras obstétricas, uma maneira de serem estimuladas para não desanimarem:

O incentivo é fundamental. Eles me incentivaram e me motivaram a fazer isso (referindo-se aos exercícios físicos). Em momento nenhum me deixaram desanimar. Quando eu já achava que não estava mais aguentando, eles me animavam. São todos muito atenciosos. 0 carinho é fora de série. [...] (Ent.11).

Nesse depoimento, percebemos a importância do apoio profissional à parturiente, favorecendo um sentimento de estímulo em relação ao trabalho de parto. A vivência que a mulher tem da parturição pode ser prazerosa ou traumática, dependendo de sua maturidade e de experiências pessoais ou familiares anteriores, e até daquelas diretamente relacionadas ao sistema de saúde, como a assistência recebida no pré-natal e durante o parto.

Estudos sobre o apoio por uma única pessoa durante 0 parto mostraram que o suporte físico e empático contínuo durante o trabalho de parto resulta em benefícios, como a diminuição de sua duração, no uso de medicações e analgesia, de partos operatórios e depressão neonatal.

Também foi evidenciado pelas mulheres que, ao terem a presença do acompanhante, sentiram tranquilidade e força para darem a luz:
[...] o que ajudou também, muito, foi quando o pai dele chegou. Também me tranquilizou bastante. [...] Ele me deu mais força, mais segurança, não que minha irmã, minha sobrinha não desse, mas quando ele chegou, sabe... Ele nasceu rapidinho (Ent. 01).

A força relatada pelas depoentes está associada à percepção de que esta prática fornece apoio na hora do trabalho de parto:

Acho que pra gente é melhor ter o apoio da família (Ent.10).

Como já discutido anteriormente, a presença e o apoio de uma pessoa conhecida pode trazer benefícios à mulher, como maior tranquilidade e força no momento do parto. Uma pesquisa sobre os efeitos psicossociais em mulheres que foram acompanhadas, recebendo apoio durante 0 trabalho de parto e no pós-parto, independente de quem oferecesse este apoio, revelou uma diminuição na ansiedade e depressão neste período. $^{16}$

Outro efeito percebido por elas foi com a movimentação corporal que contribuiu para que se sentissem mais encorajadas:

Eu achei positivo (a deambulação) até porque me deu mais coragem, né? (Ent. 02).

A mulher deve ser encorajada a atuar como sujeito do evento, pois ela tem o direito de participar das decisões sobre o nascimento. Ressalta-se, porém, que as próprias mulheres ainda têm dificuldades em assumir um papel participativo no trabalho de parto em função da cultura de subordinação a que estão submetidas. Dessa forma, para humanizar o atendimento ao nascimento, é necessário conscientizá-las, discutindo quais as suas necessidades ou demandas, pois somente assim poderão reivindicar um cuidado melhor. ${ }^{17}$

Além da coragem, evidenciamos que a mulher que decidiu pela movimentação corporal percebeu-se livre:

[...] você ter a liberdade de fazer o que quiser. De andar, de ficar em pé, sentada, ir ao banheiro, eu me sentia mais incomodada na hora que as contrações vinham. Então, eu sentava no banheiro, no vaso. Essa liberdade de ir e vir, muito boa, é muito boa mesmo (Ent. 06).

Essa liberdade sentida ao movimentar-se levou a um aprendizado em relação ao seu próprio corpo:

[...] a gente aprendeu a se tocar, saber o que está acontecendo com o teu corpo [...] (Ent. 04). 
Algumas práticas, como os exercícios de relaxamento, permitem que as mulheres reconheçam partes do próprio corpo e suas sensações, principalmente as diferenças entre relaxamento e contração, assim como as melhores posições para relaxar durante o parto. Portanto, despertar na mulher uma sensação de maior liberdade no uso dos seus movimentos corporais contribui para o seu resgate como protagonista do seu parto. ${ }^{18}$

\section{CONSIDERAÇÕES FINAIS}

Tomando como base os achados do estudo, podemos concluir que a abordagem carinhosa, a movimentação corporal e a presença de um acompanhante foram reconhecidas pelas mulheres pesquisadas como práticas e atitudes de cuidado próprias das enfermeiras obstétricas.

0 uso dessas práticas e atitudes teve efeitos considerados benéficos pelas próprias mulheres. Elas relataram ter sentido seus potenciais internos fortalecidos para a tomada de suas próprias decisões, o que significou para elas o respeito e reconhecimento de seu direito de fazer suas escolhas.

Também foram identificadas as práticas das enfermeiras da Casa de Parto como decisivas para não desanimarem durante o trabalho de parto. Isso significa que a enfermeira obstétrica é um agente estratégico no estímulo ao parto normal, particularmente quando sua prática humanística é potencializada por modelos assistências orientados pela humanização e pela utilização de práticas baseadas em evidencias científicas.

Quanto à presença do acompanhante, esta proporcionou maior tranquilidade e força, diminuindo o medo e a ansiedade delas durante o trabalho de parto, e a movimentação corporal promoveu coragem e sensação de liberdade, demonstrando que as enfermeiras reconheceram essas mulheres como sujeitas ativas e protagonistas do evento, e que adotaram as suas práticas conforme os princípios das tecnologias não invasivas de cuidado.

\section{REFERÊNCIAS}

1. Ministério da Saúde (BR). Secretaria Executiva. Núcleo Técnico da Política Nacional de Humanização. HumanizaSUS: politica nacional de humanização. Brasilia (DF);2003.

2. Ministério da Saúde (BR). Secretaria de Políticas de Saúde. Área Técnica de Saúde da Mulher. Parto, aborto e puerpério: assistência humanizada à mulher. Brasilia (DF);2001.

3. Hotimsky SN, Schraiber LB. Humanização no contexto da formação em obstetrícia. CiencSaude Colet 2005; 10(3):639-49.
4. Progianti JM, Vargens OMC. As enfermeiras obstétricas frente ao uso de tecnologias não invasivas de cuidado como estratégias na desmedicalização do parto. Esc Anna Nery Rev Enferm 2004; 8(2): 194-97.

5. Azevedo LGF. Estratégias de luta das enfermeiras obstétricas para manter o modelo desmedicalizado na Casa de Parto David Capistrano Filho. [dissertação de mestrado]. Rio de Janeiro (R): Universidade Estadual do Rio de Janeiro; 2008.

6. Merhy EE. Saúde: a cartografia do trabalho vivo. $3^{\mathrm{a}}$ ed. São Paulo(SP): Hucitec;2002.

7. Prado ML, Martins CR. Técnica, tecnologia e o cuidado de enfermagem: em busca de uma nova poética no trabalho de enfermagem. In: Prado ML, Gelbcke $\mathrm{FL}$, organizadores. Fundamentos de enfermagem. Florianópolis (SC): Cidade Futura;2002. p.19-22.

8. Nietscher EE, Leopardi MTO. 0 saber da enfermagem como tecnologia: a produção de enfermeiros brasileiros. Texto\&Contexto Enferm 2000; 9(1): 129-52.

9. Torres IA, Santos I, Vargens OMC. Construindo uma concepção de tecnologia de cuidado de enfermagem obstétrica: estudo sociopoético. Texto\&Contexto Enferm 2008; 17(4): 656-64.

10. Bardin L. Análise de conteúdo. Lisboa (PT): Ed 70; 2004.

11. BrandãoSMOC. Vivência do acolhimento da mulher encaminhada da Casa de Parto David Capistrano Filho à unidade de referência. [dissertação de mestrado]. Rio de Janeiro (R): Universidade Estadual do Rio de Janeiro; 2008.

12. Maia AR, Erdmann AL, Carraro TE, Radunz V. Princípios do cuidar. In: 0 processo de cuidar, ensinar e aprender o fenômeno das drogas: a redução das demandas. Módulo ${ }^{\circ} 4$. [curso de especialização]. Forianópolis (SC): Departamento de Enfermagem, UniversidadeFederal de Santa Catarina; 2003.

13. COSTAAA. Gênero, poder e empoderamento das mulheres. [citado 15 maio 2008]. Disponível em: http://www.agende.org.br/docs/File/dados_pesquisas/ feminismo/Empoderamento\%20-\%20Ana\%20Alice.pdf.

14. Vargens OMC, Progianti JM, SilveiraACF.0 significado de desmedicalização da assistência ao parto no hospital: análise da concepção de enfermeiras obstétricas. Rev Esc Enferm USP 2008; 42(2): 339-46.

15. Storti JPL. 0 papel do acompanhante no trabalho de parto e parto: expectativas e vivências do casal. [dissertação de mestrado]. Ribeirão Preto (SP): Universidade de São Paulo; 2004.

16. Hofemeyer GJ, Chalmers B, Wolman WL, Nikode VC, Gulmezoglu AM. Companionship in labour: do the personality characteristics of labour supporters influence their effectiveness. Curationes 1995; 18(4): 77-80.

17. Rattner D, Trench B, organizadores. Humanizando nascimentos e partos. São Paulo (SP):Senac; 2005.

18. MamedeVM,AlmeidaAM, Clapis MJ. Movimentação/deambulaçãonotrabalho de parto: uma revisão. Acta Scientiarum 2004; 26(2): 295-302

Recebido em 05/07/2009 Reapresentado em 09/03/2010 Aprovado em 10/04/2010 